\title{
Implementation of IoT Application using Geofencing Technology for Protecting Crops from Wild Animals
}

\author{
Ashwini L. Kadam1), Hoon Lee2) and Mintae Hwang3)
}

\begin{abstract}
In this paper, we propose a wild animal intrusion notification system using IoT and geofencing technology to protect crops from intruders such as wild animals. The system consists of an ultrasonic sensor to detect wild animals, LTE network communication for transmitting information about detection to a server system, geofencing technology that provides location information, and a mobile application that sends notifications to farmers in real-time. In this study, the prototype of the system was developed, and lab trials were carried out to analyze the time response performance between the hardware module and the mobile application used to receive the notification. It can be assumed that there will be no crop losses in farm fields if such a system is implemented. In the future, the system can also include wild animal intrusion prevention and prediction functions through big data analysis.
\end{abstract}

Keywords: IoT, Geofencing Technology, Intrusion Notification, Smart Farm, Wild Animal, Mobile Application

\section{Introduction}

Across the world, farming is the backbone of all economies, and the economic growth of several countries mostly depends on crop cultivation. Farms produce foodstuff for people and several raw materials for industries. In recent times, crop destruction owing to intrusion of wild animals into farm fields is a frequent challenge to farmers all over the world. The incidents of wild animals straying into farm fields and squashing crops are on the rise, triggering an economic problem for farmers. Such intrusions by animals lead to crop damage; therefore, farmers need to be constantly on their toes to protect their crops[1]. This causes farmers a huge

Received(December 27, 2019), Review Result(1st: January 29, 2020, 2nd: March 18, 2020), Accepted(May 27, 2020)

1) (Student) 51140 Dept. of Eco-Friendly Ocean Plant FEED Engineering, Changwon National University, Changwon, Gyeongnam, Korea

email: ashwinikadam33@gmail.com

2) (Professor, Corresponding Author) 51140 Dept. of Information and Communication Engineering, Changwon National University, Changwon, Gyeongnam, Korea

email: hoony@cwnu.ac.kr

3) (Professor) 51140 Dept. of Information and Communication Engineering, Changwon National University, Changwon, Gyeongnam, Korea

email: professorhwang@gmail.com 
loss of crops and resources required for expanding crops. Therefore, the prevention of animal aggression is most critical for the protection of crops. This problem can be addressed with the aid of a WSN (Wireless sensor network). Together with the IoT (Internet of Things), the sensor network lets the farmers stay connected to their farms anytime, anywhere. Emerging IoT technology with IoT devices can be used to capture wild animal detection and environmental information for human observations through the smartphone application. The IoT enhances things to be detected and/or remotely controlled through the current network infrastructure, generating opportunities for more direct interaction with the physical environment through computer-based systems and contributing towards precision, performance, and economic benefits.

Through this study, we propose a solution to overcome this problem. IoT plays a vital role in smart farming, which is an evolving model for the reason that IoT sensors are proficient in delivering data about their farming fields. This study intends to create an application with the use of developing technology, i.e. IoT and smart farming using the notification system for protecting crops. We demonstrate a connected farm aimed at providing an adequate environment for the protection of crops from wild animals based on IoT systems and geofencing technologies. We have developed a mobile application to allow farmers to track wild animal intrusion remotely. This system offers a technology that transmits data from farm fields to the cloud in real time and then to a mobile application, using which farmers can access data about intrusions from anywhere. The main objective of this study is to develop a real-time notification service on a mobile application to aid farmers. The advent of technology has led to IoT implementation using geofencing technology. The proposed novel system is cost-efficient and smart because it has its own brain in the form of Arduino Uno and is a mobile application.

The rest of the paper is organized as follows: in Section 2, we analyze the state of the art techniques and research related to crop monitoring and geofencing. In Section 3, we displayed the configuration of the proposed system and the operation flowchart. We described the prototype implementation of the proposed system in Section 4. In Section 5, we present an analysis of the performance evaluation. We conclude in Section 6 with a discussion on future research.

\section{Related Works}

Detection of wild animal intrusion is quite a challenging task. Recently, several studies have been published on crop monitoring and crop safety. Abbas et al. suggested a global positioning system (GPS) based on a location monitoring system with geofencing[2]. In their research, the 
system was able to easily track and monitor the location of the vehicle and alert it when the vehicle left the geofenced area. This system could help to reduce the incidence of vehicle thefts. It showed excellent results for the navigation and locating of vehicles with a precision of $10 \mathrm{~m}$.

Reclus and Drouard proposed geofencing for fleet \& freight management[3]. Geofencing allows remote monitoring of geographical areas encircled by virtual fences (geofences) and automatic detection when mobile objects are tracked into or out of these areas. This research discusses the basic principles of geofencing as well as some applications based on this methodology in the logistics and transportation field. Their system demonstrates that geofencing can be implemented to different fields relevant to the surveillance of people and mobile assets, as well as to other applications such as public works, civil defense, or protection.

Mobley proposed tracking and monitoring animal movements using hybrid geofencing and wireless technology and explained how networked cattle tracking system would track cattle using a base station, a mobile device and smart cattle tags[4]. This study provided a system to track cattle location and other information including temperature, movement, and blood information.

Sheshadri et al. proposed a method and/or apparatus for geofence management that can facilitate the maintenance of a geofence and deliver notifications in response to an object entering or leaving a zone bounded by a geofence[5]. This system demonstrates the implementation of a geofence; for example, an modification of a geofence may include altering the shape or size of the geofence.

Chourey et al. proposed an IoT-based wireless sensor network for the protection of crops from wild animals[6]. Wild animals also damage standing crops, which adversely affect annual crop growth, resulting in financial losses for farmers. Chourey et al.'s system implements a technical solution to help farmers protect their crops from wild animals using IoT and a WSN. It includes all types of controllers, sensors and actuators needed for WSN and has Raspberry $\mathrm{Pi}$ in the core.

Bhuvaneswari et al. proposed the design and implementation of an intelligent system for crop protection[7]. The proposed system used modernization techniques to protect crops from wild animals as well as environmental changes such as heavy rainfall and high temperature. The field is covered by a protective shield provided for the protection of crops. The benefit of the shield is that it is provided by solar panels, which also help in power generation under high temperature.

Wawrzyniak and Tomasz proposed the application of geofencing technology for spatial 
analyses in inland mobile navigation[8]. The framework outlines implementation problems related to the proximity analysis used for protection and knowledge purposes with respect to the use of geofences. This involves the generation of notifications and warning messages in the vicinity of navigational hazards. The biggest benefit of using this technology include that software developers can quickly apply it without the practical knowledge of spatial analysis.

Shubham and Vishal proposed human protection from disasters using an Android application[9]. Using this application, Android users stuck owing to natural disasters can send information about their location to their family, fire stations, and police stations and call an ambulance. The authors also created geofences that can help alert about restricted areas marked manually by a user using the app.

Sanjana et al. proposed a smart surveillance monitoring system using a Raspberry $\mathrm{Pi}$ and PIR(Passive Infrared) sensor[10]. This system extends cell phone technology to give essential protection for homes and other control applications. It is able to record/capture videos/images and transmits to a cell phone. This is beneficial because it ensures privacy and reliability.

Minwoo et al. proposed the design and implementation of a connected farm for a smart farming system[11]. The system illustrates that the farming industry could be further established by implementing new technologies such as, especially, a linked farm based on the IoT system. The authors also implemented a smartphone application that allows end users to monitor and control their connected farm remotely. This scenario holds the promise of creating an expert farm information network that inspires people to work in the agricultural sector.

\section{Configuration of the Proposed System}

In this section, we focus on the configuration and technologies used in the design and development of a mobile application for notifying farmers about the intrusion of wild animals into farm fields. [Fig. 1] illustrates the overall system configuration and its working and offers a notification service to farmers. Within the proposed system, hardware modules, namely, an ultrasonic sensor, GPS module, and LTE module are connected, and the entire procedure is conducted using Arduino Uno. Ultrasonic sensors and GPS module are used to detect targets (e.g. wild animals or humans) and to obtain the location of targets detected in the farm field respectively. The detected object and location information data are transmitted wirelessly using the LTE(Long Term Evaluation) module. The transmitted data are stored in the Firebase database and the notification about intrusion is provided to the farmer by the proposed mobile application. 
In addition, we have used geofencing technology, which works as a virtual fence to avoid notification of the farmer's entrance into the farm field. When a farmer enters a geofenced farm field with the proposed mobile application, the application will verify the farmer's location. If the location matches the geofenced farm's location, the application will terminate the notification of their entrance as an intruder for a while.

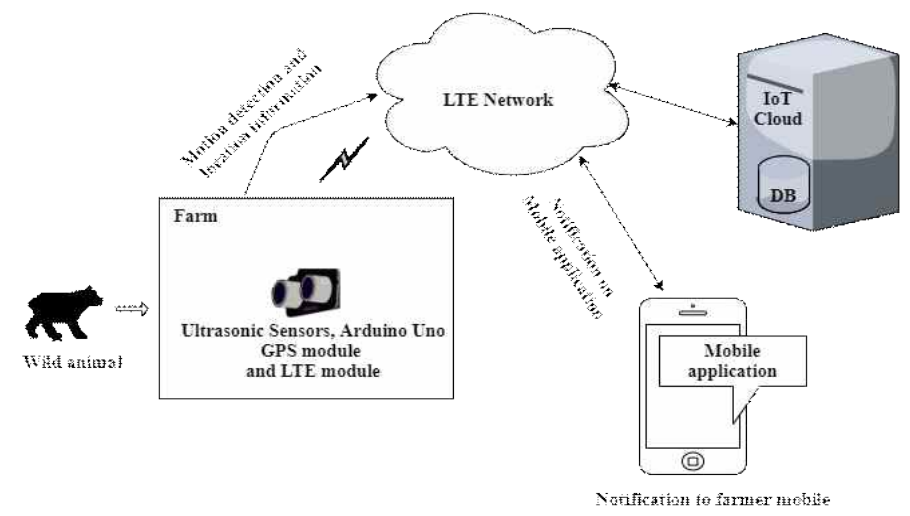

[Fig. 1] Configuration of Proposed System

[Fig. 2] depicts the process flow for the proposed system. First, the system collects the data of the object to be detected based on its motion with respect to its location and sends it to the database through the LTE. Subsequently, the mobile application verifies the location of the registered mobile number if it is within the geofence; if so, it terminates the notification. Otherwise, the farmer will receive a notification about intrusion.

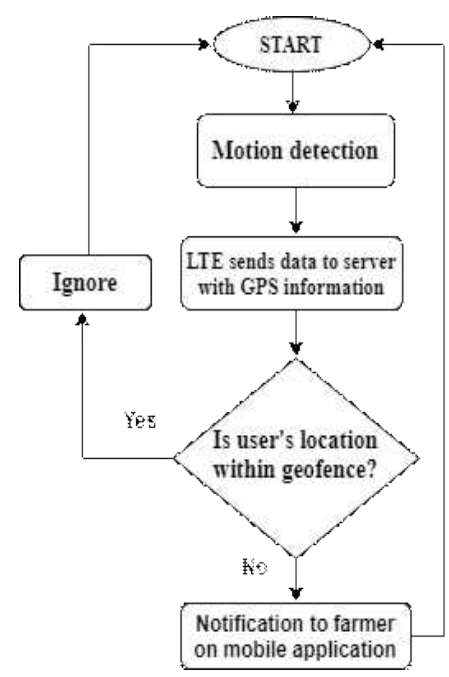

[Fig. 2] Flowchart of Proposed System 


\section{Implementation of Proposed System}

\subsection{Implementation of Hardware Prototype}

The hardware module integration of the system is presented in [Fig. 3]. The modules used in the system are as follows " 1 " is the microcontroller board Arduino Uno, "2" is an ultrasonic sensor, " 3 " is the GPS module, and " 4 " is the LTE module. Arduino Uno works at the heart of the system. The ultrasonic sensor for detection of intrusion, GPS module for location, and LTE module for wireless transmission are connected to it.

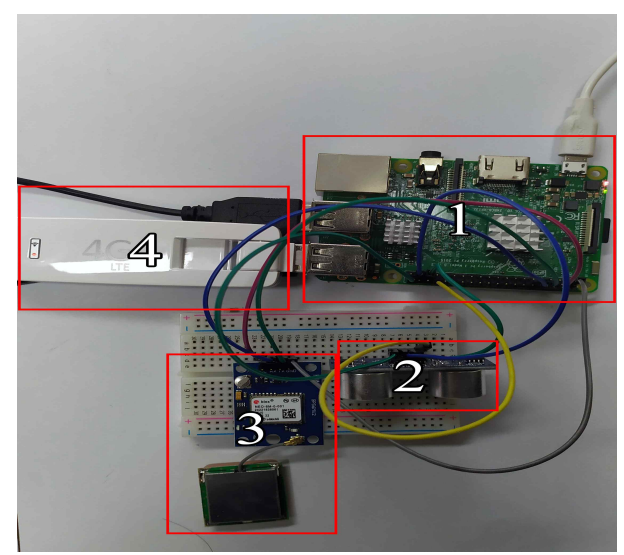

[Fig. 3] Hardware Prototype Implementation of Proposed System

The prototype of the system has been tested in the laboratory to simulate its behavior in the field. The above-mentioned hardware devices are described below in detail. The HC-SR04 ultrasonic sensor includes controller circuit, transmitter and receiver. This module detects objects within a range of $2-400 \mathrm{~cm}$. The sensor can detect motion and calculate the distance to the object in motion.

Typically, the location is determined by a GPS module. The NEO-6M GPS receiver module is applied to reliably determine the location of the GPS for the detection of intrusion by wild animals in the farm field. By interfacing a GPS module with Arduino Uno, we can monitor location in terms of latitude and longitude. The LTE modem is a prime selection, as it offers seamless connectivity, which is necessary for the proposed system. LTE networks using mobile Internet connectivity are able to upload and download faster and essentially do anything on the web. However, there are also some limitations -the LTE network cells are small; therefore 
a significantly higher number of towers are required to achieve the same coverage. This can be prohibitively expensive compared to $\mathrm{WiFi}$.

\subsection{Implementation of Software Prototype}

[Fig. 4] demonstrates the software implementation environment used for the proposed system. It consists of a database and different types of application program interfaces (APIs). The Google Maps API is used to display the map, and the Geofencing API facilitates the use of the geofence feature in the application. To provide connectivity in both APIs, we used the Google API, which communicates with the Firebase database and mobile application to store data and check the location of the application, respectively. Simultaneously, the database checks for previously stored data and if a match is identified, it will push a notification to the mobile application. Different platforms were used to deploy the API and database. The key feature of this application is that it provides real-time notification along with location about detection of wild animal intrusion near the farm field to farmers.

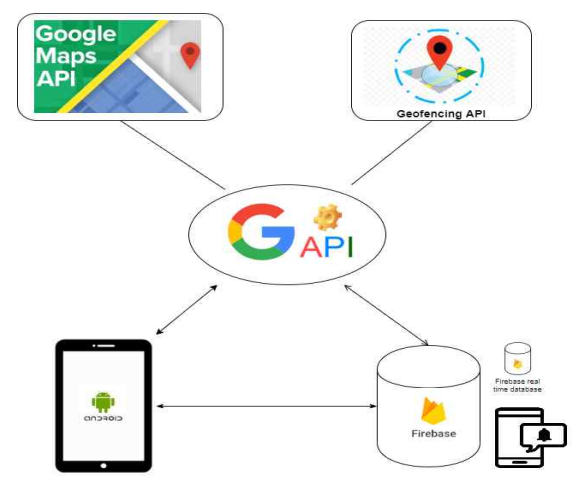

[Fig. 4] Software Implementation Environments

[Fig. 5] represents the Firebase database, which is a cloud-hosted NoSQL database that synchronizes and stores data received from the sensor and GPS module through the LTE module in real time. The data obtained from the hardware module is stored in JSON (JavaScript Object Notation) format.

The Google Maps API is used to recognize the farm field location and the Geofencing API is used to check whether the farmer is inside the farm field area, as exhibited in [Fig. 6]. Here, we assumed some parameters and mathematical functions to simulate the geofence coordinates because we assume that only one GPS module will be installed at the center of the farm field. 
The coordinates generated based on our assumptions are displayed in [Fig. 6] as A, B, C, and D. These coordinates were used to create a geofence using the Geofencing API.

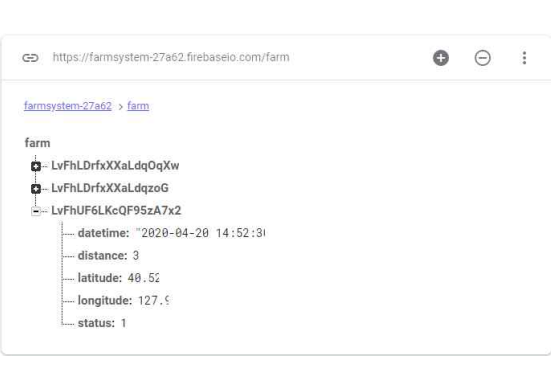

[Fig. 5] Data Stored in Database

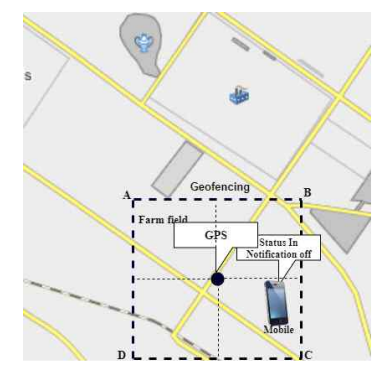

[Fig. 6] Geofencing to Farm Field

[Fig. 7] exhibits the mobile application developed using Android Studio and Java.

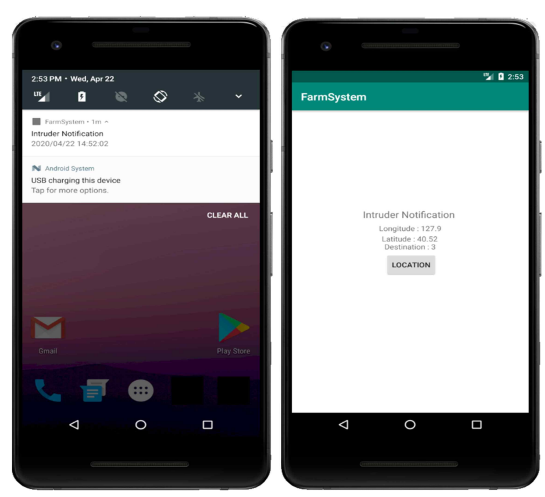

(a) Notification (b) Intruder Information

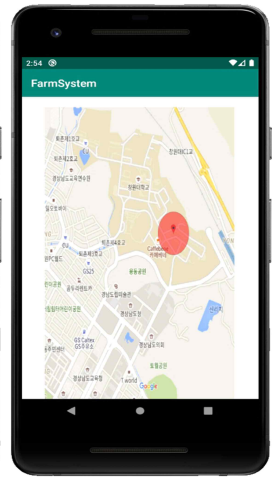

(c) Farm Field Location

[Fig. 7] Prototype of Mobile Application

The mobile application uses cloud messaging to notify the farmer about intrusions. When an intrusion is detected in the farm field, the application receives a notification, as displayed in [Fig. 7](a). By clicking on the message, the farmer can see the location as well as the distance of the intruder from the sensor, as displayed in [Fig. 7](b). The farmer can see the location of their farm field on Google Maps, as displayed in [Fig. 7](c). When they are inside the geofence, the status of the application will indicate their own location inside the geofence and no notification will be received. The mobile application provides the feature of warning the farmer beforehand by informing them about the intruder being at an appropriate location before intrusion into the farm field. 


\section{Performance Evaluation}

The purpose of the designed system is to ensure that the mobile application responds quickly to detection of intrusion. Therefore, we conducted laboratory trials to evaluate the performance of the proposed system, we analyzed the response time delay from the sensor module to the mobile application using time stamps. The results indicate that there is an average delay of $1.57 \mathrm{~s}$ in receiving a notification. This delay is acceptable and may be caused by loss of Internet connectivity.

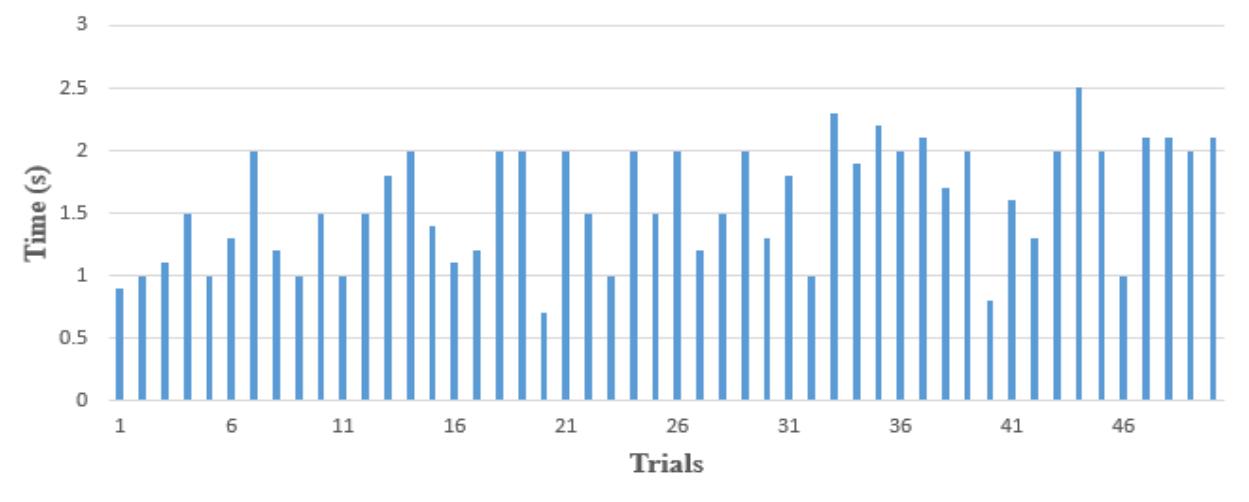

[Fig. 8] Response Time

To know the total number of transactions per second/response that occurred in a given period. For example, assume that in 1,440 seconds, a geofence has 900 transactions. Dividing total transactions in a given period by the number of seconds in the period. In the example, 800 transactions divided by 1,440 seconds equals 0.055 transactions per second/response from geofence to mobile.

\section{Conclusion}

Crop damage owing to wild animal intrusions into farm fields ultimately leads to losses for farmers. The proposed smart farming technique with IoT application developed using geofencing technology, which is effective for avoiding animal intrusion, consists of an ultrasonic sensor for detection of motion near the farm field, a database, and a mobile application. The main advantage of the proposed system is that farmers can receive real-time notifications with 
location if animal intrusion occurs in the farm field, which is one of the easiest and fastest means. The proposed system was implemented as a prototype and lab trials were performed. Based on the observed results, it can be concluded that the use of this application will ensure minimum or no loss of crops. An evaluation of the performances of the hardware module and mobile application indicates that the proposed system responds efficiently with notifications.

Further, if we apply big data analysis and higher quality hardware modules, we can improve the performance of the system. In the future, we will consider including the effect of additional climatic conditions, such as temperature and humidity, on the operation of the mobile application to provide further convenience to the farmer.

\section{Acknowledgement}

This research was financially supported by Changwon National University in 2019-2020.

\section{References}

[1] N. Srinivasa Rao, V. L. K. Chaitanya, I. Naga Sai Kiran, K. Vamshi Krishna, Smart fencing for crop field monitoring, International Journal for Research in Applied Science \& Engineering Technology, (2019), Vol.7, No.1, pp.2361-2363.

[2] A. Abbas, I. Mohammed, S. Jurdi, L. Audah, N. Alduais, GPS based location monitoring system with geo-fencing capabilities, Proceedings of the International Conference of Electrical and Electronic Engineering, (2019), June 24-25; Putrajaya, Malaysia.

[3] F. Reclus, K. Drouard, Geofencing for fleet \& freight management, International Conference on Intelligent Transport Systems Telecommunication IEEE, (2009), October 20-22; Lille, France.

[4] Mobley T., inventor; nMode Solutions Inc, assignee, Tracking and monitoring of animals with combined wireless technology and geofencing, United States patent application USA 13/917,328, (2013)

[5] Sheshadri, H. Suhas, Aditya N. Srivastava, Method and/or apparatus for geofence management, U.S.A. Patent $8,941,48$ (2015)

[6] S. R. Chourey, P. A. Amale, N. B. Bhawarkar, IOT based wireless sensor network for prevention of crops from wild animals, International Journal of Electronics, Communication and Soft Computing Science \& Engineering, (2017), Vol.5, No.1, pp.57-60.

[7] P. Bhuvaneswari, M. Bhumika, J. Navya, T. M. Nayana, Design and implementation of an intelligent system for crop protection, International Journal for Innovative Engineering and Management Research, (2019), Vol.8, No.6, pp.62-68.

[8] Wawrzyniak, Natalia, Tomasz Hyla, Application of geofencing technology for the purpose of spatial analyses in inland mobile navigation, Baltic Geodetic Congress (BGC Geomatics), (2016), June 2-4; Gdansk, Poland. 
[9] Yelne, Shubham, Vishal Kapade, Human Protection with the Disaster Management Using an Android Application, International Journal of Scientific Research in Science, Engineering and Technology, (2015), Vol.1, No.5, pp.15-19.

[10] P. Sanjana, P. Mahalakshmi, A. John Clement Sunder, R. Swathi, Smart Surveillance Monitoring System Using Raspberry $\mathrm{Pi}$ and PIR sensor, International Journal of Computer Science and Information Technologies, (2014), Vol.5, No.6, pp.7107-7109.

[11] M. Ryu, J. Yun, T. Miao, I. Y. Ahn, S. C. Choi, J. Kim, Design and implementation of a connected farm for smart farming system, Proceedings of 2015 IEEE Sensors, (2015), November 1-4; Busan, South Korea. 